\title{
Mediación pedagógica para la autonomía en la formación docente
}

\section{Pedagogical Mediation for the Autonomy of Future Professionals in Teacher Training}

\author{
Ana María Hernández Segura' \\ División de Educación Básica \\ Centro de Investigación y Docencia en Educación. \\ Universidad Nacional \\ Heredia, Costa Rica \\ anahernandez63@hotmail.com \\ Luz Emilia Flores Davis² \\ División de Educación Básica \\ Centro de Investigación y Docencia en Educación. \\ Universidad Nacional \\ Heredia, Costa Rica \\ luzdeflores@yahoo.com
}

Recibido 27 de junio de 2012 • Corregido 20 de setiembre de 2012 • Aceptado 07 de noviembre de 2012

Resumen. El presente artículo trata la importancia de la mediación pedagógica para promover el tránsito de la heteronomía a la autonomía intelectual, en la formación docente del estudiantado de primer ingreso a las carreras de Educación. Se comparten algunas estrategias y experiencias implementadas por las autoras de este artículo. Se destaca la necesidad fundamental de escuchar las voces de los estudiantes y las estudiantes $y$, de esta forma, conocer sus expectativas, opiniones y experiencias previas, punto de partida para el desarrollo de diversos temas y conceptos, lo cual favorece una participación real y libre en las dinámicas de mediación pedagógica y, por tanto, espacios para que se expresen con autonomía. Lo anterior, permite pasar de una pedagogía centrada en la enseñanza, a una pedagogía centrada en el aprendizaje, de manera que el futuro educador y educadora sean protagonistas de su formación y vayan construyendo su propia identidad intelectual, desde la acción-reflexión-acción. Se considera que el primer año de vida en la educación superior es un espacio privilegiado para fomentar el desarrollo de la autonomía en las futuras y los futuros docentes y, como lo señala Freire (2004), el respeto por la autonomía es un imperativo ético.

Palabras claves. Formación docente, autonomía, mediación pedagógica.

\footnotetext{
Máster en Educación con mención en Docencia Universitaria, Licenciada en Educación Preescolar de la Universidad Nacional, Costa Rica y Terapista de lenguaje, Madrid, España. Actualmente se desempeña como docente, investigadora y extensionista en la División de Educación Básica del Centro de Investigación y Docencia en Educación (CIDE) de la Universidad Nacional. Estudiante del Doctorado Latinoamericano de la Universidad Estatal a Distancia, Costa Rica.

2 Doctora en Educación con mención en Mediación Pedagógica. Máster en Educación Superior. Catedrática universitaria. Ha ocupado los cargos de Directora de Docencia, Directora de Desarrollo Profesional y Cooperación Universitaria, Presidenta de la Junta de Becas y Coordinadora de la Comisión Técnica de Admisión de la Universidad Nacional. Actualmente, se desempeña como investigadora, extensionista y docente en la División de Educación Básica del Centro de Investigación y Docencia en Educación (CIDE) de la Universidad Nacional, Costa Rica y docente en la Maestría en Danza con mención en Formación Dancística del Centro de Investigación, Docencia y Extensión Artística (CIDEA), Universidad Nacional, Costa Rica.
} 
URL: http://www.una.ac.cr/educare

\begin{abstract}
This article studies the importance of pedagogical mediation in helping first-year students in the Teacher Training area to advance from intellectual heteronomy to intellectual autonomy. It explains some strategies and experiences implemented by the authors. It focuses on the need of paying attention to students in order to understand their expectations, opinions and previous experiences as a basis for developing different topics and concepts, to favor an actual free participation in the pedagogical mediation dynamics and, consequently, opportunities for students to express themselves with autonomy. The aforementioned strategies allow for the transition from a pedagogy centered on teaching to a pedagogy centered on learning, in such a way that the future educator becomes a protagonist in his/her formation and constructs his/her own intellectual identity, based on the concept of action-reflection-action. The authors of this article consider that the first year in higher education is a privileged opportunity to develop the autonomy of future teachers, and, as stated by Freire (2004), the respect for autonomy is an ethical imperative.
\end{abstract}

Keywords. Teacher training, autonomy, pedagogical mediation.

La División de Educación Básica (DEB) del Centro de Investigación y Docencia en Educación (CIDE), de la Universidad Nacional, como heredera de la Escuela Normal de Costa Rica en el ámbito de formación de educadoras y educadores, y como unidad académica de una universidad que aspira a formar profesionales conscientes de las necesidades de la sociedad y del mundo en que viven, forma docentes en las siguientes carreras de grado: Pedagogía con énfasis en I y II Ciclos, Pedagogía con énfasis en Educación Preescolar, Pedagogía con énfasis en Educación Especial y Pedagogía con énfasis en I y II Ciclos en Inglés.

Además, a nivel de posgrado, la DEB ofrece la Maestría en Pedagogía con mención en Atención a la Diversidad en los Procesos Educativos, y la Maestría en Pedagogía con mención en Desarrollo y Atención Integral a la Primera Infancia.

Las autoras de este artículo somos académicas que ofrecemos cursos en las diversas carreras de la DEB y participamos en un proyecto de extensión universitaria cuyo propósito es el mejoramiento de los procesos iniciales de lectura y escritura con niños, niñas, jóvenes y personas adultas. Nuestro interés se centra en la formación de formadoras o formadores, sea en los procesos formales conducentes a un título, o en la actualización o capacitación de docentes y otras personas interesadas en promover la lectura y escritura.

Quienes ejercemos la docencia realizamos la práctica pedagógica según las propias creencias, las cuales provienen de nuestra historia de vida, de los principios que compartimos y de las experiencias realizadas. Enseñamos como creemos que se aprende, y muchas veces ese concepto está relacionado con el estilo personal de aprender y con la manera como nos han enseñado. Señala Day (2006) que: 
Las identidades profesionales de los docentes -qué y quiénes son, su autoimagen, los significados que se vinculan a sí mismos y a su trabajo, y los significados que le atribuyen a otros- están por tanto, asociados a la materia que enseñan (...), sus relaciones con los alumnos, sus papeles y las conexiones entre éstos y su vida fuera de la escuela. (p. 68)

En este sentido, es innegable el impacto de la identidad de algunas o algunos docentes y su modelo de enseñanza en el ejercicio de la docencia y en las vidas. Para Contreras (1999), la autonomía del profesorado tiene que ver con esa búsqueda y aprendizaje continuo de la reconstrucción de su propia identidad profesional. En una reciente investigación, Barahona (2011) concluye que "uno de los factores de mayor influencia para el desarrollo de la autonomía ha sido el relacionado con los rasgos de personalidad de los maestros y, en general, el tipo de relación pedagógica establecida en los procesos educativos (p. 123).

\section{El punto de partida de futuros y futuras docentes}

El estudiantado de primer ingreso a la universidad proviene de un sistema educativo que, en términos generales, forma para la heteronomía, es decir, para responder a las exigencias del mismo sistema mediante exámenes centrados en los contenidos de las disciplinas científicas. Esto hace necesario que la vivencia educativa de las futuras y los futuros educadores en la universidad, sea coherente con los principios del modelo pedagógico institucional.

Así, en nuestra experiencia de formación de formadores y formadoras, entendemos que el estudiante o la estudiante universitaria está aprendiendo cómo aprender a lo largo de su vida, a cómo mantenerse actualizada o actualizado de los nuevos conocimientos y a cómo aprender de su propia práctica docente. De ahí la importancia de concebir los espacios de aprendizaje como contextos y ambientes que permitan las vivencias personalizadas de aprender a aprender (Assmann, 2002).

Con el interés de conocer, mediante un diagnóstico de entrada, a estudiantes de nuevo ingreso, en el curso Evolución de la Educación Preescolar de la Carrera de Pedagogía con énfasis en Educación Preescolar de la Universidad Nacional (Hernández, 2012), se aplicó un instrumento en la primera sesión del primer ciclo del 2012, a 31 estudiantes, de los cuales treinta son mujeres y uno es varón.

El instrumento tiene un total de 9 preguntas y frases incompletas. La primera parte está relacionada con aspectos del curso: ¿Qué significa para usted educación preescolar? ¿Por qué eligió está carrera? ¿Cuál es el papel de la educación Preescolar en el desarrollo del niño y la niña? Y la segunda versa sobre sus expectativas en relación con el curso y otros temas relacionados, tales como: Yo aprendo mejor cuando... Un clima o ambiente de aula apropiado para aprender es aquel que... Yo espero de la profesora... Yo espero de este curso... Lo que yo puedo aportar para el éxito de este curso es... 
Así, a partir de ese instrumento, el estudiantado tiene el espacio para externar algunos motivos por los cuales quieren ser educadores o educadoras. Al respecto, entre sus repuestas destacan las siguientes expresiones: "Me siento capaz y con habilidades para ejercer esta profesión", "Se requieren muchas habilidades que creo tener", "Me di cuenta que era mi vocación", "Sé que soy capaz de trabajar con los niños", "Es una profesión que siempre me ha llamado la atención y me sorprende el mundo de los niños y su manera de ver la vida", "Es lo que de verdad me apasiona hacer", "Me interesa la educación y me sentiría cómoda al trabajar con niños", "Es mi vocación, es lo que quiero hacer", "Porque es la carrera que quiero", "Me apasionan los niños y su capacidad de aprendizaje", "Me gustaría ayudar a los niños, para que sepan cosas para la vida", "Me apasiona y es algo que siempre he soñado".

Sobresale en esas respuestas un gran entusiasmo y convicción en cuanto a que esta es la carrera en la que desean formarse; de igual manera se percibe esa pasión por el reto que implica ser educador o educadora. Al respecto, Day (2006) menciona lo importante que es incluir las emociones y los sentimientos para que la enseñanza y el aprendizaje sean de calidad, ya que permiten dar sentido a las relaciones con el mundo. Agrega el autor citado que la pasión es relevante en el campo educativo, ya que"(...) sin la pasión por la asignatura y por el alumno, es difícil mantener los niveles de planificación, interacción inteligente y compromiso emocional necesarios para mantener el título de un "buen" docente" (p. 51).

Por lo anterior, es un reto, para quienes tenemos la responsabilidad de formar al futuro personal docente, mantener y enriquecer a lo largo de su proceso de formación esas expectativas, emociones y pasiones por la carrera elegida.

Sobre las expectativas de este grupo de primer ingreso sobre el curso y sus docentes, las autoras de este artículo, mediante ese diagnóstico, hemos recogido evidencias en las cuales expresan que esperan un profesor o profesora que sea respetuosa, con buen trato, amigable y amable, a la vez que les brinde apoyo, paciencia, comprensión y motivación. Además, que exprese y demuestre su conocimiento y profesionalismo; que brinde buenas explicaciones y aclare dudas; que dé lo mejor de sí, y que enseñe para la vida.

Estas expectativas tienen relación con los nuevos escenarios educativos, como lo menciona Assmann (2002): "El nuevo encanto y el nuevo placer de la educación requiere la unión entre sensibilidad social y eficiencia pedagógica" (p. 32). Enfatiza en ese compromiso ético del educador o educadora con la excelencia pedagógica y con la construcción de un clima esperanzador en el ámbito escolar. Así lo ilustran las siguientes expresiones del grupo de la muestra con respecto a lo que esperan de sus profesores y profesoras universitarias: "Que sea amable y que me enseñe muchas herramientas que necesito para esta profesión", "que sea compresiva, pero a la vez un poco estricta para que así desarrollemos mucha responsabilidad en este curso", "aprender mucho en todo momento y en un ambiente agradable", "adquirir muchos conocimientos y no solo memorizar algo", "que sea exigente y me ayude a dar lo mejor de mí para ser una excelente profesional". 
Respecto a las expectativas sobre el curso, 22 estudiantes mencionan que esperan que sea provechoso, que les permita aprender mucho, y que les dé buenas bases para la carrera; 5 que sea de ayuda para su formación profesional, y otras respuestas hacen referencia a aspectos tales como: que puedan disfrutarlo, que haya un ambiente agradable, y que sea claro. Algunas de las expresiones que ilustran estas expectativas son las siguientes: "ser el mejor de mi área", y"enamorarme cada día de esta profesión", "aprender bastante y no solo para los exámenes","que sea de ayuda para mi formación profesional", "para ser una gran maestra en un futuro". De nuevo emergen retos en el campo de la docencia universitaria, en el sentido de lograr ese"enamoramiento" por la carrera elegida y, en especial, por contribuir con la formación de excelentes educadoras y educadores.

\section{El reto de la formación de docentes autónomos o autónomas en los escenarios actuales}

Un proceso formativo que busque transitar de la heteronomía intelectual hacia la autonomía requiere generar acciones para que sus aprendientes formulen sus propias preguntas y para que vayan construyendo las respuestas desde la acción-reflexión-acción, lo cual les abrirá caminos hacia nuevas interrogantes. Esto favorecerá el abandono de la búsqueda de aprobación externa y la búsqueda y expresión, con entera libertad, de sus propios puntos de vista, sus convicciones y sus inquietudes.

Sobre el proceso de formación docente, Sanjurjo (2009) hace alusión a tres enfoques.

El tradicional en el cual prevalece una formación lineal y tradicional basada en la imitación. En este enfoque se visualiza al docente como un operario o técnico que solamente aplica acríticamente la teoría.

El segundo enfoque es el hermenéutico-reflexivo, donde prevalece una relación dialéctica entre la teoría y la práctica, y en el cual los sujetos son mediados por las acciones que realizan. Es decir, se concibe la formación docente como un complejo proceso de mediación que realiza el docente cuando se está formando, que le va permitir sustentar su práctica. Agrega Sanjurjo (2009), que desde este enfoque se enfatiza la interpretación y comprensión del significado de las acciones:

La interpretación de las acciones ayuda a revelar el significado de las formas particulares de la vida social, y con ello contribuye a la comprensión del sentido de las acciones para los propios actores, quienes si se reconocen en esa interpretación, podrán modificarlas. (p. 18)

El tercer enfoque, que denomina crítico, permite situar y reconocer la complejidad e influencia del contexto político, económico, cultural y personal en las prácticas educativas, que son a su vez construcciones sociales. Para Sanjurjo (2009), "la teoría crítica articula la reflexión a los problemas de valores e intereses sociales" (p. 25). 
Avanzar hacia los enfoques hermenéuticos-reflexivos y críticos, que promuevan esa autonomía intelectual, es lo que pretendemos al solicitar al estudiantado de reciente ingreso a la universidad, que asista al menos una vez por semana a una institución educativa; en un primer momento a observar la dinámica institucional y de clases $y$, posteriormente, a realizar intervenciones pedagógicas con niños y niñas.

Desde los planes de estudio de las carreras de la División de Educación Básica, se otorga gran importancia al área de lectura y escritura; así, en la carrera de Pedagogía con énfasis en Educación Preescolar, se ofrece un curso que tiene como eje la alfabetización, en el sentido amplio del término que implica la capacidad de la persona de hablar, leer, escribir y pensar en forma crítica y creativa. En la carrera de Pedagogía con énfasis en I y II Ciclos, se brindan dos cursos para la formación en el área de lectura y escritura.

Estos cursos, en ambas carreras, están ubicados en el primer nivel del plan de estudios con estudiantes que recién ingresan a la universidad. Además comparten el enfoque pedagógico, y profundizan el conocimiento y análisis de diferentes corrientes innovadoras para la enseñanza y aprendizaje de la lectura y la escritura; además, enfatizan la relación pensamiento lenguaje, a partir de una concepción de la lectura y escritura desde su función social.

Por otra parte, requiere la incorporación de la estudiante o del estudiante una vez por semana en centros educativos, con el propósito de que observen, analicen, reflexionen y promuevan procesos de alfabetización inicial desde el constructivismo. Lo anterior, porque la mediación pedagógica del aprendizaje de la lectura y escritura implica el conocimiento de los procesos de construcción que se realizan según los niveles evolutivos y los contextos sociales; su estudio permite, al estudiantado, ahondar en la utilización de alternativas metodológicas y generar sus propias propuestas para compartir tanto con niños y niñas, como con personas adultas.

Como bien lo expresa Freire (2004), "(...) el respeto a la autonomía y a la dignidad de cada uno es un imperativo ético y no un favor que podemos o no concedernos unos a los otros (...) (p. 28).

La pretensión de trasladarse de una pedagogía centrada en la enseñanza a una centrada en el aprendizaje tiene que ver con descentrar los espacios de poder del profesor o profesora y propiciar los procesos de autoevaluación crítica del estudiantado, tarea nada fácil en una sociedad que privilegia los productos sobre los procesos y donde las conductas son valoradas desde afuera de la persona y desde los reglamentos, más que desde la ética.

En la medida en que nos evaluamos y conocemos, nos acercamos más al autoconocimiento, percatándonos de la libertad que disponemos o de la capacidad que poseemos para tomar decisiones libres, para dialogar e intercambiar ideas, con un estilo propio, maximizando el potencial analítico y creativo con el que contamos, fortaleciendo los valores y principios sociales a los que responde el comportamiento. (De Luca, 2009, p. 909) 
Es indispensable que quienes aprenden asuman el protagonismo en su formación y, para ello, la mediación pedagógica debe favorecer relaciones democráticas y respetuosas centradas en el diálogo y en la valoración de las capacidades del estudiantado. Por lo anterior, las observaciones que se realizan en la práctica preescolar y escolar deben ser compartidas en el aula universitaria para realizar un análisis crítico-propositivo.

La autonomía se desarrolla en contextos relacionales. De esta manera, en los procesos formativos tiene que ver con las interacciones mediador o mediadora -aprendiente, y entre pares. “(...) Sólo bajo una concepción no impositiva de la enseñanza puede entenderse la aspiración a una autonomía que se construye en la relación (De Luca, 2009, p. 905). En esta misma línea, Contreras (1999) señala que la autonomía no es un atributo o capacidad que se posee, se trata más bien de una construcción permanente en una práctica de relaciones, "(...) la autonomía se actualiza y se configura en el mismo intercambio que constituye la relación" (Contreras, 1999, p. 150). Para este autor, la autonomía no es una característica del individuo, sino un modo deseable de relación social, basada en la colaboración y entendimiento y no en la imposición.

Se trata de una autonomía, que descansa en un principio ético de una razón descentrada del sujeto y centrada en la intersubjetividad lingüística y el reconocimiento recíproco entre sujetos, basada en principios universalistas y aplicados con responsabilidad por afectividad, sensibilidad y conciencia frente a las situaciones de "sufrimiento" humano y de injusticia compartidas con los "otros" (...). (Pérez, 2012, p.17)

El desarrollo de la autonomía está en íntima relación con las posibilidades de participación real, con la construcción de criticidad y con la libertad de expresión y acción responsable. Tiene que ver con la capacidad de asumir los retos de realidades cambiantes e inciertas. La teoría que se estudia descontextualizada difícilmente puede promover cambios. "El propio discurso teórico, necesario a la reflexión crítica, tiene que ser de tal manera concreto que casi se confunda con la práctica (...)" (Freire, 2004, p. 19).

Como lo indica Negrin,(2003), generalmente en la formación docente existe una separación entre la fundamentación teórica que se estudia en la universidad y la práctica pedagógica.

Los alumnos se acercan a las prácticas de la enseñanza después de un proceso específico de formación que les ha ido permitiendo visiones fragmentarias, construidas muchas veces a partir de enfoques necesariamente parciales e inacabados, pero que al no reconocerse como tales obturan toda posibilidad de problematización, en tanto el sujeto debe incluirse en esa verdad absoluta desde una posición de posesión de ese saber y no de interrogación. (Negrin, 2003, p. 308) 
La superación de la distancia entre el espacio universitario y las instituciones preescolares y escolares pasa por el reconocimiento del profesionalismo docente y requiere la promoción del diálogo, de la capacidad de escuchar y aprender de las demás personas.

Este contacto con las aulas escolares y preescolares desde el inicio de la carrera, de los estudiantes y las estudiantes de primer ingreso, es valorado por ellos y ellas mediante el cuestionario de evaluación final del curso antes mencionado. Fue contestado por 24 estudiantes. Sobre la frase: "Considero que las visitas realizadas a las diferentes modalidades de atención a niños y niñas...", sobresalen las siguientes expresiones que permiten conocer el significado particular de esa vivencia para cada estudiante: "Ha sido una forma de mirar las realidades que acontecen en la sociedad costarricense", "Siento que valió mucho la pena, ya que a partir de esas visitas he podido confirmar mucho de lo que nos explican en clase", "Me permiten acercarme a la vida diaria y así estar más segura de lo que realmente quiero", "Me ayudó a reafirmar que esta es la carrera que más deseo desempeñar, me motiva a formarme mejor para ser una educadora de calidad", "Fue algo inolvidable, algunas fueron lindas otras no, lo hacen reflexionar a uno", "Me ha ayudado a tener contacto con la realidad de la educación en Costa Rica", "Me ha ayudado a saber qué es lo que realmente se vive al tratar con niños y niñas", "Me ayudó a darme cuenta de que no quiero cambiarme de carrera, sino más bien a amar lo que voy a realizar", "Se aprende muchísimo con las visitas", "Que este país en la parte de educación debe mejorar y quiero hacer ese aporte", "Me dejó grandes experiencias y aprendizajes", “Es una experiencia única”.

Estas declaraciones reafirman lo anotado anteriormente, respecto a que la autonomía tiene relación directa con la participación real, porque abre el espacio para que los futuros y las futuras docentes sean protagonistas de su propio proceso de formación. Además, este tipo de experiencia fuera del aula universitaria resulta coherente con los principios metodológicos de los planes de estudio de la DEB, sobre la "funcionalidad de los aprendizajes", el cual permite al estudiantado una relación práctica-teoría-práctica y, por tanto, un proceso de formación con sentido. Dicho principio señala que "(...) la funcionalidad implica el traslado del aprendizaje y de la formación a los contextos prácticos (...)" (División de Educación Básica, Centro de Investigación y Docencia en Educación, Universidad Nacional, 2010, p. 53).

\section{Fusionando puentes de aprendizajes}

Desde el proyecto de extensión "Aprendizaje de la lectoescritura" se invita al profesorado queatiende las secciones dondelos grupos estudiantiles universitarios realizan sus observaciones e intervenciones pedagógicas a participar en una serie de talleres en la Universidad. El propósito de esta actividad es agradecerle su apertura, al aceptar en sus aulas a las futuras y los futuros docentes, así como también de propiciar el mejoramiento de la mediación pedagógica. Este espacio de compartir experiencias resulta de suma importancia para disminuir la brecha entre lo que se plantea en la universidad y lo que se realiza en las aulas preescolares y escolares. 
Para los maestros y maestras en servicio, la participación en los talleres de la universidad les permite reflexionar acerca de sus prácticas pedagógicas, intercambiar experiencias entre pares y ponerse al día en nuevas corrientes e investigaciones educativas, en este caso en lo referente al tema de la lectoescritura.

Desde los talleres hemos detectado algunas necesidades o ausencias en el campo de mediación pedagógica de los procesos de lectura y escritura, por ejemplo, la mayoría de participantes carecen de fundamentación teórica, continúan utilizando métodos superados desde hace décadas, muestran deficiencia y ausencia de formación en este campo, buscan recetas, métodos y desconocen los procesos evolutivos de la lectura y escritura.

En estos espacios de socialización entre colegas de diferentes instituciones educativas, las cuales tienen sus propias historias, rutinas y su cultura, es donde pueden poner en crisis sus perspectivas y trascender hasta la reflexión de sus valores y prácticas sin estar limitados o aislados en el espacio del aquí y ahora de la vida del aula escolar (Contreras, 1999).

Es evidente que la sociedad demanda que la educación y, por tanto, el personal docente de respuestas a los problemas complejos y diversos que la aquejan, lo que puede generar, en este cuerpo, angustia y soledad en una tarea tan importante. Es así como el grupo de docentes participantes en estos talleres manifiesta su necesidad de apoyo y acompañamiento en su labor, para muchos y muchas realizada en contextos de marginalidad y pobreza extrema, lo cual la problematiza más.

Además, en los talleres, los docentes y las docentes tienen la libertad de externar sus inquietudes y expresar sus voces de lo que viven cotidianamente en el ejercicio de su profesión. Por ejemplo, mencionan la necesidad de:"Cambiar los programas, pero sobre todo la evaluación, para que haya más espacios donde se fomente la lectura", "Que nos den el espacio y libertad para trabajar la lectoescritura en el nivel preescolar", "Se debe dedicar más tiempo a la lectoescritura en el aula", "Cantidad de niños en el aula y tiempo tan limitado por lecciones", "Falta de apoyo en el hogar, padres analfabetos", "Contexto pobre de lectoescritura en que están inmersos los niños", "Capacitarme más, actualizarme”, entre otros.

Es pertinente mencionar las experiencias y aprendizajes que han logrado construir y vivenciar los docentes y las docentes en estos talleres; entre algunas, indican que han podido conocer que el aprendizaje de la lectura y escritura es un proceso y que tiene diversos niveles de conceptualización para llegar al nivel convencional; que se les han generado conflictos en forma positiva, ya que se les ha permitido la reflexión para revalorar o mejorar su práctica docente; reconocen la importancia su necesidad de actualizarse en temas tan relevantes como la lectura y la escritura; manifiestan reiteradamente la necesidad de actualización profesional, y que no se debe temer el cambio, sino luchar para no caer en clases magistrales y aburridas.

En la formación de formadores o formadoras, una vez determinados los propósitos pedagógicos, debe tomarse conciencia del papel mediador en la construcción de aprendizajes. Por ello, en nuestro caso, promovemos que tanto quienes participan en los talleres, como el 
estudiantado universitario, encuentren sentido a lo que aprenden y, por lo tanto, también asuman que su mediación pedagógica debe estar atenta al sentido o sentidos que, desde sus contextos, las niñas, niños, jóvenes o personas adultas otorgan a los nuevos conocimientos.

La diversidad de realidades en que se inscribe la labor docente hace indispensable la utilización de una gran variedad de estrategias de mediación pedagógica. Resulta, entonces, comprensible que la utilización de métodos para enseñar a leer y escribir haya dado paso a propuestas abiertas capaces de atender la complejidad del aprendizaje y los contextos donde se llevan a cabo.

Como requisito de la mediación pedagógica es necesario partir de los conocimientos previos, lo cual implica conocer qué piensa la persona aprendiente respecto a la temática o asunto en estudio, y tiene que ver con la comprensión del vocabulario que se utiliza para entablar una verdadera comunicación. Pero también involucra conocer las experiencias vividas, las cuales siempre conllevan subjetividades y sentires.

Los planes de estudio de la División de Educación Básica, Centro de Investigación y Docencia en Educación, Universidad Nacional (2010), tienen, entre sus principios metodológicos, la enseñanza constructiva, lo que implica considerar, como punto de partida del proceso de aprendizaje, los conocimientos y experiencias previas de los estudiantes y las estudiantes. Otro principio hace referencia a la significatividad de los aprendizajes y propone buscar relaciones entre las construcciones previas de las aprendientes y los aprendientes, y los temas o conceptos por desarrollar en el curso.

Como una forma de concretar en la práctica del aula universitaria estos principios metodológicos, desde los cursos de lectura y escritura se le pide al estudiantado, durante las primeras sesiones de clase, que prepare un "póster" o afiche, básicamente con imágenes, donde plasmen la respuesta a la siguiente pregunta: “Cómo aprendí a leer y escribir?".

Las experiencias generadas a partir de esta consigna han permitido vivenciar en el aula universitaria esa significatividad y construcción de aprendizajes, ya que el grado de emoción y motivación que implica la elaboración de este material les lleva a realizar una mirada retrospectiva a ese momento, en el que muchas veces requirieron la ayuda y participación de sus madres, padres, hermanas o hermanos, como actores partícipes de tan importante acontecimiento.

Así lo evidencia las siguientes frases externadas por estudiantes y recolectadas por las autoras de este artículo: "Aprendí con la ayuda de mi familia, cuando mis hermanas mayores hacían sus tareas, yo salía corriendo a buscar un cuaderno y un lápiz para tratar de imitar lo que hacían", "Mi familia fue muy importante en este proceso, porque siempre se mostraban dispuestos a ayudarme", "Recuerdo que mi mamá me ayudaba, se sentaba conmigo y ella me apoyaba", "Aprendí a leer mis dibujos, yo era consciente que lo que yo leía no era lo que la gente acostumbraba a leer, pero lo mío era propio, era mi forma de aprender, lo mismo era con la escritura, no era más que un poco de garabatos", "Aprendí con la ayuda de los cuentos que me leían cuando era niña, al preguntar qué estaba escrito en los carteles o rótulos", "Antes de 
entrar a la escuela pasaba en una competencia con mi hermano probando quién leía primero los anuncios en los establecimientos públicos", "Aprendí a leer con mi mamá, primero me enseñó a escribir el nombre desde que estaba en el kínder y desde que tenía la curiosidad de leer y escribir todo", "Yo aprendí a leer y a escribir con la ayuda brindada por mi mamá, mi papá y mi abuelita que me ponían a leer el periódico".

Lo expuesto anteriormente lleva a reflexionar acerca de la importancia de que las futuras y futuros educadores tengan la vivencia de estrategias de construcción de puentes cognitivos y emocionales entre sus experiencias previas y el tema a desarrollar; ello les da la oportunidad de ser protagonistas de su proceso de formación, con la autonomía y libertad para expresarse creativamente.

\section{Conclusión}

La pedagogía tiene relaciones permanentes con otras ciencias, no obstante posee un campo de conocimiento propio que el educador o la educadora debe asumir para desarrollar una praxis autónoma.

El desarrollo de la autonomía en las futuras y los futuros docentes tiene que ver con el estar satisfechos y satisfechas con la elección de la profesión; con la capacidad de tomar decisiones independientes que les permitan el desarrollo de su propia creatividad, así como la de sus aprendientes con quienes comparten; con la seguridad profesional y la capacidad de investigar, y con la coherencia entre los planteamientos conceptuales y la praxis universitaria.

El primer año de vida en la educación superior constituye un espacio privilegiado para fomentar el desarrollo de la autonomía en las futuras y los futuros docentes, lo cual requiere un esfuerzo sostenido en el resto de la formación profesional y aún después de ella.

La vivencia de una formación universitaria en el diálogo, la cooperación y la libertad abre las puertas a educadoras y educadores creativos. Cuando se acrecienta la autonomía, entendida en un marco de relaciones respetuosas y solidarias, y se promueve una lectura más compleja de las realidades, se camina en la búsqueda de sociedades más equitativas.

\section{Referencias}

Assmann, H. (2002). Placer y ternura en la educación. Hacia una sociedad aprendiente. Madrid: Narcea.

Barahona, H. (2011). Relación entre la autonomía y las experiencias educativas previas de los actores curriculares encontradas en un contexto de educación superior de Bogotá, Colombia. Entramado, 7(1), 114-127. Recuperado de http://redalyc.uaemex.mx/src/inicio/ ArtPdfRed.jsp?iCve $=265420116008$ 
URL: http://www.una.ac.cr/educare

Contreras, J. (1999). La autonomía del profesorado (2ª ed.). Madrid: Ediciones Nárcea.

Day, C. (2006). Pasión por enseñar. La identidad personal y profesional del docente y sus valores (2a reimp.). Madrid: Nárcea.

De Luca, C. (septiembre, 2009). Implicaciones de la formación en la autonomía del estudiante universitario. Electronic Journal of Research In Educational Psychology, 7(18), 901-922. Recuperado de http://www.investigacion-psicopedagogica.org/revista/new/ ContadorArticulo.php?325

División de Educación Básica, Centro de Investigación y Docencia en Educación, Universidad Nacional. (2010). Plan de Estudios. Heredia, Costa Rica: Universidad Nacional.

Freire, P. (2004). Pedagogía de la autonomía. Saberes necesarios para la práctica educativa. Sao Paulo: Paz e Terra. Recuperado de http://webdelprofesor.ula.ve/nucleotachira/oscarg/ materias/epistemologia/lecturas/freire.pdf

Hernández, A. (2012). Curso: Evolución de la Educación Preescolar. Licenciatura en Pedagogía con énfasis en Preescolar. Universidad Nacional de Costa Rica. Centro de Investigación y Docencia en Educación. División de Educación Básica.

Negrin, M. (diciembre, 2003). Saberes teóricos y práctica docente: Razones de un desencuentro. Educación, Lenguaje y Sociedad, 1(1), 307-312. Recuperado de http://www.biblioteca. unlpam.edu.ar/pubpdf/ieles/n01a19negrin.pdf

Pérez, L. (2012). Autonomía, solidaridad y reconocimiento intersubjetivo. Claves éticas para políticas sociales contemporáneas. Revista de Estudios Sociales, 42, 13-26. Recuperado de http://res.uniandes.edu.co/view.php/760/index.php?id=760

Sanjurjo, L. (2009). Los dispositivos para la formación en las prácticas profesionales. Argentina: Homo Sapiens Ediciones.

\section{Cómo citar este artículo, según APA:}

Hernández, A. M. y Flores, L. E. (2012). Mediación pedagógica para la autonomía en la formación docente Revista Electrónica Educare, 16(3), 37-48. Consultado de http://www.revistas.una.ac.cr/index.php/EDUCARE/issue/current

Nota: Para citar este artículo en otros sistemas puede consultar el hipervínculo "Como citar el artículo" en la barra derecha de nuestro sitio web:

http://www.revistas.una.ac.cr/index.php/EDUCARE/index 